\title{
1 Efficiency of fisheries is increasing at the ecosystem level
}

\author{
2 Nis S Jacobsen ${ }^{1,2}$, Matthew G Burgess ${ }^{3,4,5}$ \& Ken H Andersen ${ }^{1}$ \\ ${ }^{1}$ Centre for Ocean Life, DTU Aqua, Charlottenlund Castle, Charlottenlund 2920, Denmark; ${ }^{2}$ School of Aquatic and \\ Fisheries Science, University of Washington, Seattle, WA 98195, USA; ${ }^{3}$ Sustainable Fisheries Group, University of \\ California, Santa Barbara, CA 93106, USA; ${ }^{4}$ Marine Science Institute, University of California, Santa Barbara, CA \\ 93106, USA; ${ }^{5}$ Bren School of Environmental Science and Management University of California, Santa Barbara, CA \\ 93106, USA
}

\begin{abstract}
Managing fisheries presents trade-offs between objectives, for example yields, profits, minimizing ecosystem impact, that have to be weighed against one another. These trade-offs are compounded by interacting species and fisheries at the ecosystem level. Weighing objectives becomes increasingly challenging when managers have to consider opposing objectives from different stakeholders. An alternative to weighing incomparable and conflicting objectives is to focus on win-wins until Pareto efficiency is achieved: a state from which it is impossible to improve with respect to any objective without regressing at least one other. We investigate the ecosystem-level efficiency of fisheries in five large marine ecosystems (LMEs) with respect to yield and an aggregate measure of ecosystem impact using a novel calibration of size-based ecosystem models. We estimate that fishing patterns in three LMEs (North Sea, Barents Sea and Benguela Current) are nearly efficient with respect to long-term yield and ecosystem impact and that efficiency has improved over the last 30 years. In two LMEs (Baltic Sea and North East US Continental 4 Shelf), fishing is inefficient and win-wins remain available. We additionally examine the efficiency of North Sea and Baltic Sea fisheries with respect to economic rent and ecosystem impact, finding both to be inefficient but steadily improving. Our results suggest the following: (i) a broad and encouraging trend towards ecosystem-level efficiency of fisheries; (ii) that ecosystem-scale win-wins, especially with respect to conservation and profits, may still be common; and (iii) single-species assessment approaches may overestimate the availability of win-wins by failing to account for trade-offs across interacting species.
\end{abstract}

Keywords Ecosystem-based fisheries management, efficiency frontiers, Pareto efficiency, size-spectrum
Correspondence:

Nis S Jacobsen,

School of Aquatic

and Fisheries Science,

1122 NE Boat St,

Seattle, WA 98105 ,

USA

Tel.: +1 (206) 519-

2349

3 Fax: XXXXXXXXXX

E-mail:

nisjac@uw.edu

Received 1 Mar

2016

Accepted 6 Jun 2016

Introduction 
Calibration

Yield and ecosystem state

Economic rent and ecosystem state

Discussion

Moving towards the frontier

Models and calibration

Applications and Conclusions

Acknowledgements

References

Supporting Information

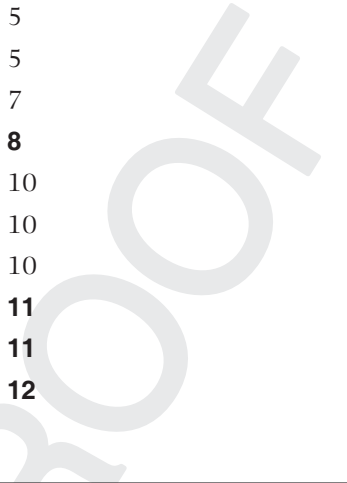

\section{Introduction}

Ecosystem-based fisheries management (EBFM) mandates an accountancy of direct and indirect effects of fishing on marine populations (Pikitch et al. 2004). Although this has been recognized for many years, implementation of EBFM has been slow (Skern-Mauritzen et al. 2015), and terms of reference for multispecies management are largely unresolved, despite recent progress (Patrick and Link 2015). In seeking to define terms of reference for an EBFM, one of the central challenges is defining the objectives.

Like most resource management problems, fisheries management has myriad objectives. Most of these fall within the 'triple bottom line' of economic, ecological and social objectives (Halpern et al. 2013), which are challenging to weigh against one another because there are trade-offs, that is improvement on one objective may come at the cost of another. Single-species management often avoids this challenge by targeting maximum sustainable yield (MSY) or maximum economic yield (MEY), which lead to biomass depletions that are widely perceived as acceptable (Hilborn et al. 2015). However, the complexity of trade-offs is significantly amplified in multispecies frameworks (Link 2002; Pikitch et al. 2004; Andersen et al. 2015a), and this has sometimes led to conflicting management advice. For example, some studies have recommended reducing fishing pressure on forage fish to protect yields of valuable predator species (e.g. Smith et al. 2011), while other studies (sometimes using the same models) have recommended increasing fishing pressure on forage fish to boost yields and maintain the ecosystem structure (e.g. Garcia et al. (2012); see Rice and Duplisea (2014) for a review of this debate).
Without needing to weigh different objectives against one another, scientists and managers can target Pareto efficiency - a state from which it is impossible to improve with respect to any objective without regressing with respect to at least one other. Trade-offs between objectives are then measured by the efficiency frontier - the set of all possible outcomes that are Pareto efficient (see Polasky et al. (2005, 2008); Lester et al. (2010, 2013); White et al. (2012); Halpern et al. (2013); Rassweiler et al. (2014) for examples of such analyses in various resource management contexts). If the current state of a particular ecosystem is found to be inefficient with respect to its objectives, then it is possible to improve on one or more simultaneously (e.g. Polasky et al.(2008)), leading to a 'win-win' situation that can be relatively uncontroversially targeted by management (Carpenter et al. 2009).

Efficiency frontier frameworks have become increasingly common in quantifying trade-offs in marine spatial planning (Lester et al. 2010, 2013; White et al. 2012; Halpern et al. 2013; Rassweiler et al. 2014), but have only sporadically been used to quantify trade-offs between broad fisheries management objectives at the scale of large marine ecosystems (LME) (Cheung and Sumaila 2008). Here, we quantify trade-offs among yield, profit and ecosystem conservation objectives in five LMEs bordering three continents: the North Sea, the Baltic Sea, the Barents Sea, the Benguela Current and the Northeast US Continental Shelf (NEUSCS) (Fig. 1). To this end, we develop a novel calibration method for size-spectrum models that allows us to explore the effect of fishing different parts of the ecosystems. We use the calibrated models to simulate the efficiency frontier for each system and show how ecosystem exploitation patterns in 
(a)

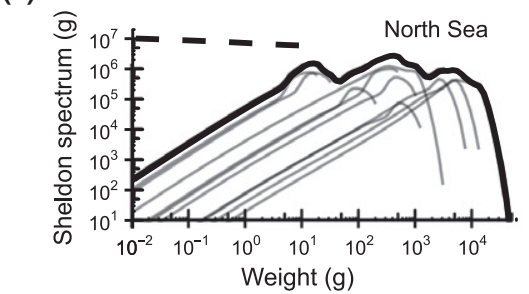

(c)

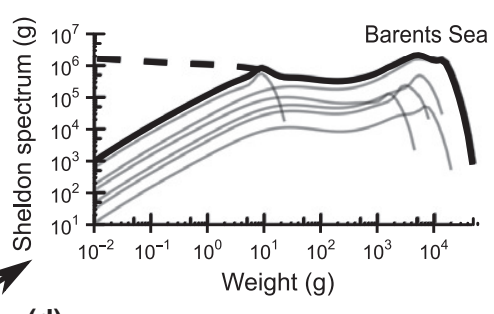

(d)
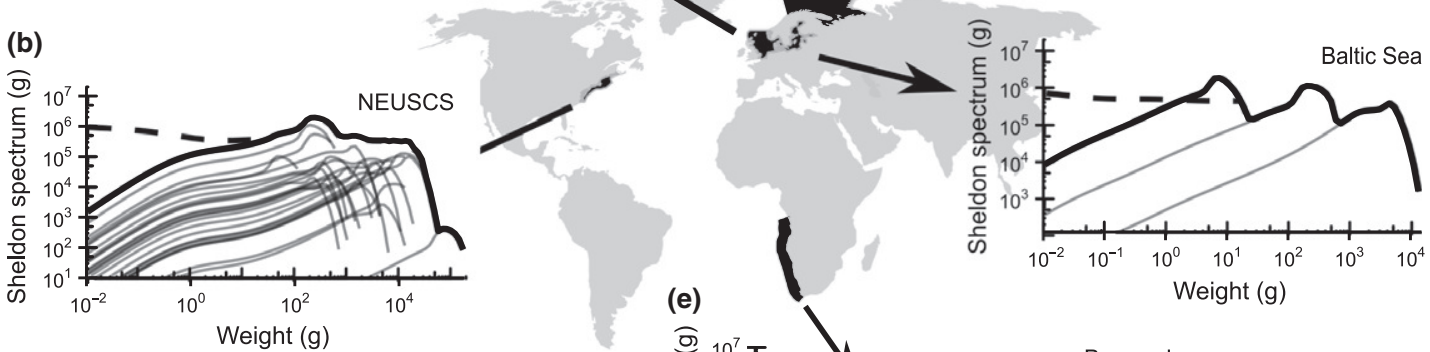

(e)

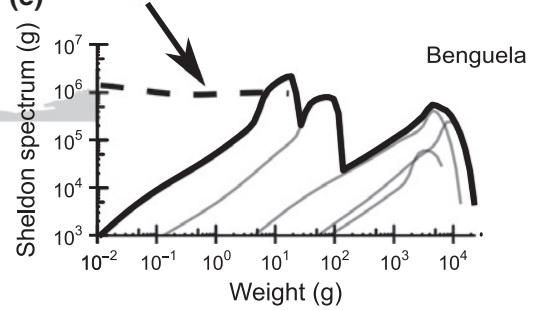

Figure 1 The five modelled large marine ecosystems (a) the North Sea, (b) North East US Continental Shelf, (c) Barents Sea, (d) Baltic Sea and (e) Benguela Current with their predicted Sheldon spectra from the calibrated models in equilibrium (corresponding to average spawning biomass over the period 1992-2002).

most cases seem to be approaching the frontier. Additionally, we project the exploitation patterns required to reach the frontier and compare them to the most recent ones.

\section{Methods}

\section{Ecological models}

We use size-spectrum models to calculate the efficiency frontiers in the considered LMEs. Size-spectrum models are based on individual-level processes and therefore have the advantage that most of the parameters can be derived from metabolic theory (Brown et al. 2004) or cross-species analysis (Hartvig et al. 2011). The models are based on a combination of the process of big individuals eating smaller ones (Ursin 1973) leading to predation mortality on prey and available energy for predators, and a bioenergetic submodel that links the available energy for growth and reproduction to the asymptotic size of predator species. Specifically, we apply the size-spectrum framework from Hartvig et al. (2011) and reviewed in Andersen et al. (2015b), while we use the RAM stock assessment database to calibrate the models to observed biomass distributions of commercially exploited fish stocks (Ricard et al. 2011). Stocks that are not commercially exploited as well as lower trophic level species are included as a background spectrum that provides additional food (up to $20 \mathrm{~g}$ ). The background spectrum is not included in the calculation of the total yield and the impact of fishing. For full model description and calibrations, see Appendices A, B, C and D.

We use LMEs as the study areas to create the models. LMEs are potentially large enough to justify ignoring migration effects for most species, while at the same time having sufficient spatial overlap between species to model interactions. We focus on a five different LMEs (Fig. 1): the North Sea, the Baltic Sea, the Barents Sea, the Benguela Current and the Northeast US Continental Shelf (NEUSCS). The LMEs represent different attributes, that is few species (Baltic Sea), high latitude (Barents Sea), high-exploitation temperate system (North Sea), upwelling (Benguela Current) and species-rich (NEUSCS). These systems were chosen as representatives as the number of stock 
assessments within them are sufficient to perform meaningful multispecies calibrations. In this respect, they represent sample of exploited ecosystems biased towards fully exploited, well-managed ecosystems.

We calibrate the models to average spawning biomass and fishing mortality in the period 19922002. This period is chosen as it is covered by most assessments (with limited gaps) from the LMEs. We validate the models by comparing projected biomass, mortality, growth and catches to observations from the calibration time period (Figures D1-D5) and by looking at the temporal spawner biomass distributions 10 years outside the time period (i.e. 10 years before and 10 years after (Figures C1-C6)). The biomass size distributions under equilibrium are visualized as 'Sheldon spectra', which are proportional to a histogram of biomasses in log widths (Fig. 1).

\section{Ecological indicator}

We use a custom indicator of ecosystem impact of fishing, denoted $I$, that aggregates a measure of depletion of all species relative to their unfished abundance. The unfished abundance is calculated at equilibrium by setting $F=0$ for all species in the system. The goal is to describe community structure and diversity relatively to the unfished community, which is recognized to be important for ecosystem services and function (Odum et al. 1971; Cardinale et al. 2012). The indicator is increasingly penalised when any species drops below 20\% of its unfished spawner biomass (Fig. 2):

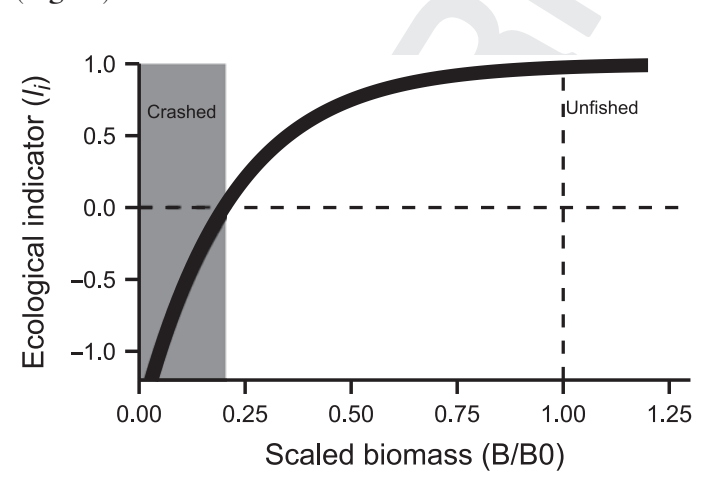

Figure 2 The indicator used to assess the state of a single species; the ecological indicator is calculated by averaging over all species. The indicator is scaled such that $I_{i}$ becomes negative when spawning stock biomass $B_{i}$ is less than $20 \%$ (dotted horizontal line) of the unexploited biomass, $B_{i, 0}$ (dashed vertical line).

$$
I=\frac{1}{n} \sum_{i} 1-\tau^{\frac{-B_{F, i}}{B_{0, i}}-0.2}
$$

where $\tau$ is a parameter determining the sensitivity of $I$ to depletion. $B_{F, i}$ is the spawner biomass of species $i$ in the fished scenario and $B_{0, i}$ is the spawner biomass in the unfished scenario, and $n \mathbf{5}$ is the total number of species. The parameter $I$ is largest in undisturbed systems, that is when $B_{F}=B_{0}$ for all species, and thereby provides an index measure of ecological state; large values of $I$ are interpreted in our framework as better ecological outcomes. Qualitative results concerning efficiency are robust towards changes in $\tau$ (Appendix G); we hereafter use $\tau=100$.

\section{Economic model}

We additionally use a simple economic model to calculate the rent of the fisheries in the North Sea and the Baltic Sea (Andersen et al. 2015a), based on prices in the Danish fishery. The model calculates resource rent, $r_{i}$ of the ith species as

$$
r_{i}=\int_{0}^{W_{\infty, i}} Y_{i}(w) p(w) d w-C_{i}
$$

where $p(w)$ is the price per weight, defined as $p$ $(w)=a_{p} W^{C}$ and the cost is $C_{i}=a_{c} F_{0} W_{\infty, i}^{b} . Y_{i}$ is the yield and $W_{\infty, i}$ the asymptotic weight. The parameter $a_{c}$ is scaled such that the fishery operation under the equilibrium used for fitting is marginally profitable (Figure E1).

\section{Fishing}

We model fishing assuming trawl selectivity for each species, where fish are gradually selected with a $50 \%$ selection at $0.1 W_{\infty}$ for all species. We calculate the exploitation needed to reach maximum sustainable yield $\left(F_{\mathrm{MSY}}\right)$ for each species by iteratively changing the input fishing mortality until maximum yield is reached under equilibrium. The other species in the systems are fished with a constant mortality during this calculation. We test the impact of these simplifying assumptions by comparing the simulated $F_{\text {MSY }}$ with the ones estimated in the stock assessments (Appendix D).

To estimate the efficiency frontiers, we model fishing as a combination of three fleets in each LME. The fleets target small, medium or large fish (see Table F1). The fleets correspond roughly to a 'forage fishery' (small, $W_{\infty}<1500 \mathrm{~g}$ ), a 'pelagic 
fishery' (medium, $1500<W_{\infty}<10000 \mathrm{~g}$ ) and a 'demersal fishery' (large, $W_{\infty}>10000 \mathrm{~g}$ ) (Andersen et al. 2015a). By calculating all combinations of fishing mortalities in the three fleets (upper bound of $2 F_{\mathrm{MSY}, \mathrm{i}}$ ), we characterize the trade-off spaces between total yield and $I$, and total rent (profit) and $I$ across the ecosystems. The efficiency frontier for each set of objectives (yield-ecological indicator $(I)$, profit-ecological indicator) is the set of equilibrium outcomes beyond which it is impossible to improve on one objective without regressing on the other. We use an equilibrium-based measure of efficiency instead of a transient measure, because it is possible to transiently have combinations of yield or profit and ecosystem state that are impossible to achieve at equilibrium (because they are unsustainable, e.g. when starting from a high abundance and employing a high fishing mortality). We find transient yield-ecosystem state combinations outside the equilibrium efficiency frontier in the Barents Sea, for example (Fig. 3). Defining efficiency in reference to these unsustainable outcomes would be misleading.

The efficiency of a particular fishing pattern is evaluated by comparing the outcomes it would produce with all other possible outcomes. Here, we present the fishing patterns that are Pareto efficient (Fig. 3). We also quantify hind-casted yields, profits and ecological indicator ( $I$ )-values from 1980 to 2010 by simulating the ecosystems forced by observed fishing patterns (Fig. 3).

We measure trends in efficiency (with respect to conservation and yields or profits) by comparing: (i) the equilibrium state that would have resulted from the average fishing mortality from 1980 to 1985 in perpetuity (the value is averaged in the beginning to avoid sensitivity to developing fisheries at that time period) and (ii) the equilibrium state that would result from fishing mortalities in most recent management (year 2010) in perpetuity. Comparing these equilibrium states measures both the direction and magnitude of the trend in fishing patterns within the ecosystem state-yield/ profit trade-off spaces (Fig. 3).

\section{Results}

\section{Calibration}

The calibrated models predict the average spawning biomass distributions in the five LMEs accurately (Figures D1-5ab). The emergent growth rates of individuals correspond closely to the observations (Figures D1-5b), but with some exceptions, for example in the North Sea the modelled growth is marginally slower than observed growth (Figure D7). Other observed rates (natural mortality and $F_{\text {MSY }}$ ) also show patterns close to the observed ones, although with some variation among the LMEs.

The temporal trends (both within and outside the calibrated time period) correspond strikingly well to the estimated biomass trend from the assessments, considering that the only external driver is changes in fishing mortality (Figures C1-6). For some species, smaller species in particular, fluctuations are not fully captured in the models (see e.g. Benguela Current anchovy, Engraulis capensis, Engraulidae). This is to be expected as fluctuations in shorter lived species are driven partly by environmental variability that is not resolved by the model.

\section{Yield and ecosystem state}

We estimate that the fisheries in the North Sea, the Benguela Current and the Barents Sea LMEs were operating close to the efficiency frontier with respect to yield and ecological state (Fig. 3, a, c, d). Over the 1980-2010 period, changes in fishing patterns in each of the three LMEs led to a reduction in long-term ecological impact by our measure $(I)$ at the expense of a decrease in long-term yield (Fig. 3). Fishing patterns in the North Sea and Barents Sea did not change in their average distance from the long-term efficiency frontier, whereas fishing patterns in the Benguela Current moved closer to the frontier - implying projected improvements in both yield and ecosystem state (I).

Conversely, fishery outcomes in the North East US Continental Shelf and the Baltic Sea were inefficient with respect to yield and ecological state. We project that these LMEs have potential to increase yield over twofold without negatively impacting ecological state (by our metric, I). They could also improve I significantly (from $\approx 0.55$ to $\approx 0.90$ ) without compromising yield. The increase in $I$ in these two systems can be achieved by rebuilding stocks with lower exploitation rates than is employed in most recent year (Fig. 4). In the last year, considered all fleets (small, medium and large) are overfishing and cause some species to go below $20 \%$ of 

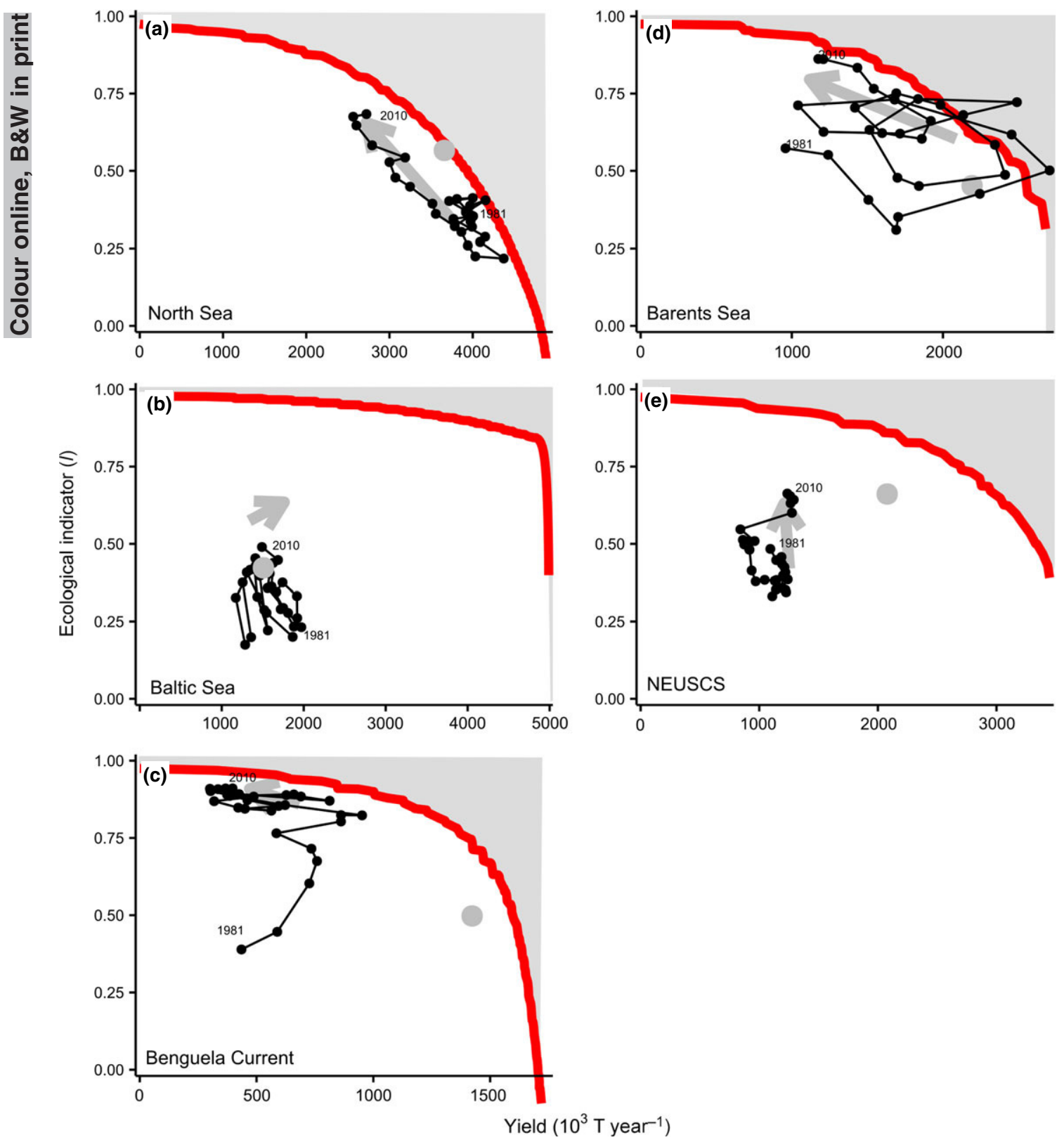

Figure 3 Yield and ecology state efficiency frontiers of five large marine ecosystems. The red line is the frontier and grey point represents all species fished at maximum sustainable yield for each species individually. The black line with dots shows the temporal movement of the systems (from 1980 to 2010). The grey arrow denotes the change in management from 1980-1985 to 2010 by calculating the equilibrium solutions using the average fishing mortalities from those years, respectively. Grey shaded area is the trade-off area that not attainable in the long term; systems may enter this area, but only in a transient.

the unfished spawning biomass which increasingly penalizes $I$. The observed temporal changes in fishing patterns in both systems would result in minor changes in equilibrium yield, with some improvement of ecological state in the NEUSCS (Fig. 3b, e).
The fishing mortality that leads to maximum sustainable yield for each species individually (single-species management) is only efficient with respect to yield and ecological state in the North Sea, although it is an improvement over the 2010 fishing pattern in the NEUSCS (Fig. 3). 

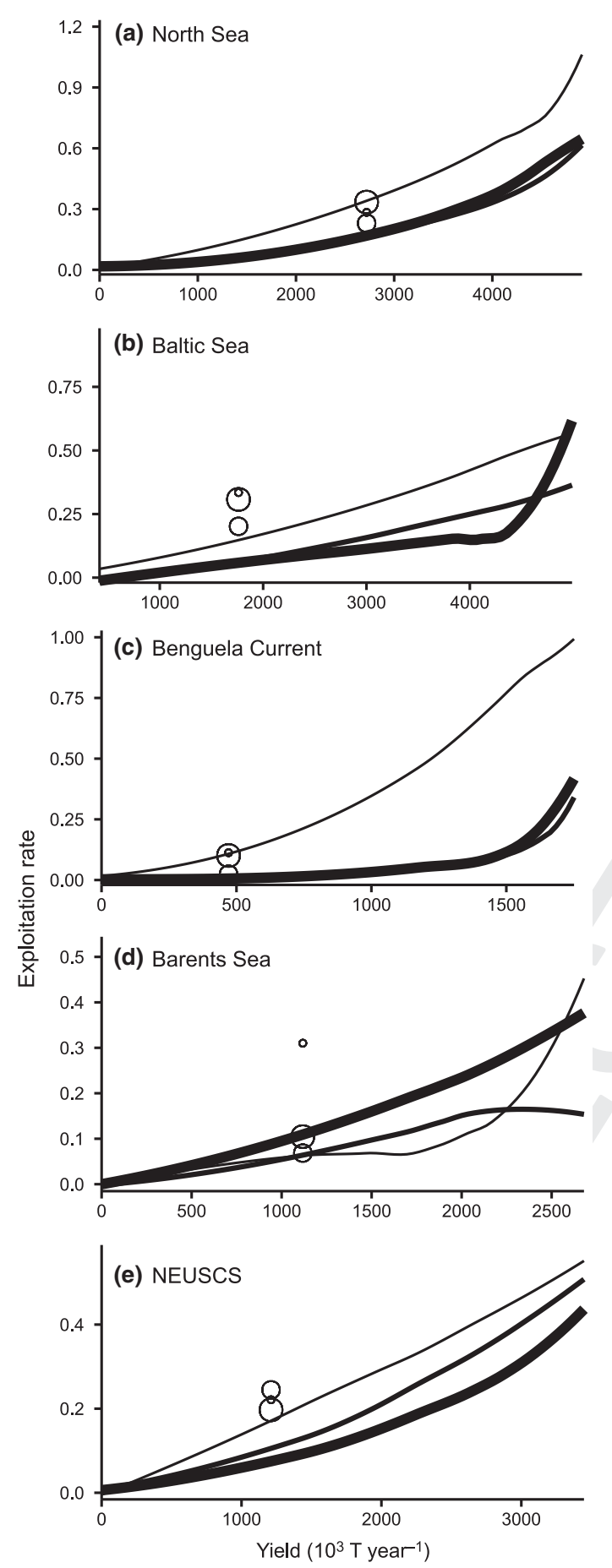

Figure 4 Exploitation rate (mean yield per biomass, yield/biomass, for small, large and medium species) at the efficiency frontiers as a function of yield (1 mill. metric tonnes per year). The lines are smoothed by a loess function. Line thickness indicates fleets targeting small, medium or large species. 2010 exploitation pressure and total yield in equilibrium is denoted by the open circles (small, medium, large, for small, medium and large fleets, respectively).
The exploitation patterns required to reach the efficiency frontier involve exploitation of all species included in the analysis (Fig. 4). The fishing pattern at the frontier varies among LMEs, particularly related to the exploitation of the small species: in most systems small species should be more highly exploited to reach the frontier. This is not the case in the Barents Sea, however, where the small species (capelin, Mallotus villosus, Osmeridae) is less tolerant to depletion, possibly due to being the dominant prey fish included in the model. The highest yields on the efficiency frontier in four of five systems (all except NEUSCS) are achieved by employing a high fishing mortality on the large species and utilizing the release of predation on smaller species, which increases their productivity.

All of the ecosystems could maintain their current yield and increase $I$ by redistributing their exploitation patterns (Figs 3 and 4); or alternatively increase yield without further structural changes to the ecosystem (Fig. 3). The North Sea has the potential to move closer to the frontier with a slight increase in the exploitation of small fish and a slight decrease in the exploitation of large species. (Fig. 4a, circles vs. lines). Efficiency gains are achievable in the Baltic Sea and NEUSCS by decreasing exploitation of all three fleets which achieves the same total yield while increasing I (Fig. 4a and e). In the Benguela Current, yields could be kept constant with less effort directed towards large species. The Barents Sea has potential to increase $I$ without compromising yield by lowering the fishing mortality of small species.

\section{Economic rent and ecosystem state}

In examining the efficiency frontiers for the North Sea and Baltic Sea with respect to economic rent instead of yield, we focus on the years 20002010, as the price data used for the model are from this decade (Fig. 5). We project that both systems have a scope for $\approx 30 \%$ rent increase using a more rent-oriented distribution of fishing mortalities. This emphasizes the difference between maximizing biomass yields and economic yields: The North Sea was performing close to the yield-efficiency frontier in this time period, whereas the economy-efficiency frontier has ample scope for improvement. However, we also find evidence for steady improvement in the efficiency of the fishing 


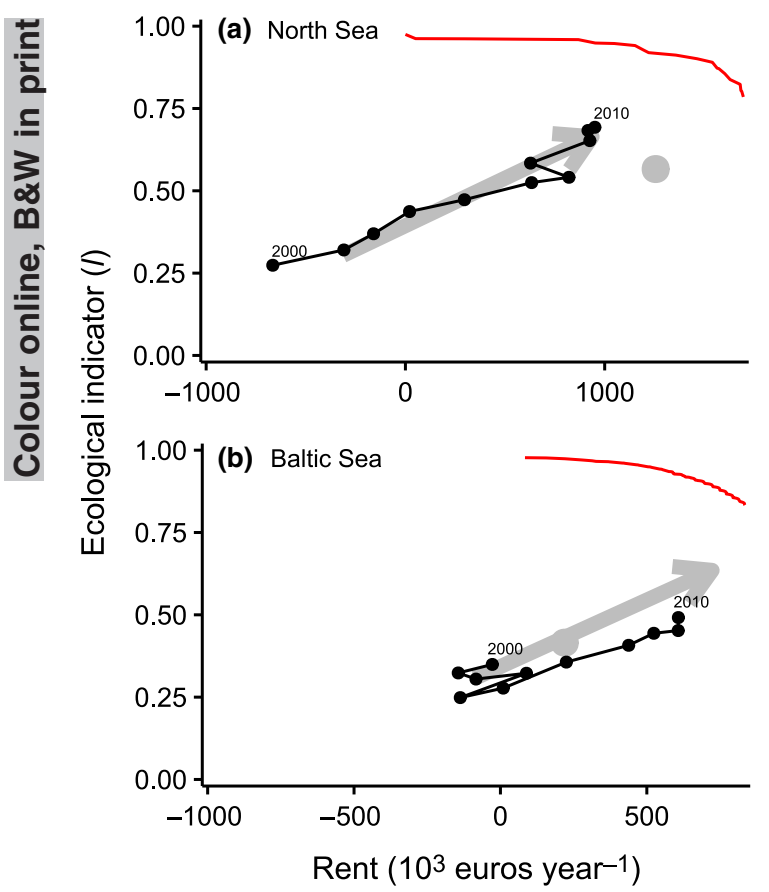

Figure 5 The economic efficiency frontier of the North Sea and the Baltic Sea. The red line is the efficiency frontier between 2000 and 2010. The grey arrow shows the average direction since year 2000; both systems have increased their total rent, while improving ecological state.

patterns in these LMEs over the last decade, which we predict to increase both their total rent as well and our indicator $(I)$ of ecosystem state.

Single-species MSY management targets would not be efficient with respect to rent and ecosystem state in either of these two LMEs. In principle, this is not surprising: MSY management targets yield and neither profits nor ecosystem state per se. In a single-species model where $B_{\mathrm{MSY}}<B_{\mathrm{MEY}}$, MSY management would also not be efficient with respect to profits and ecological state (by our measure, I). However, the degree of inefficiency of MSY management with respect to profits and $I$ is noteworthy (Fig. 5), by showing a large potential for win-wins in both systems. MSY management is inefficient because less fishing effort is required to reach the economic frontier (Fig. 6) than the yield frontier (Fig. 4). An increase in fishing effort is additionally included in the cost function (eq. 2 ), and thus, the higher fishing mortality produces an increase in costs for fishing operations. This is similar to the single-species case (where generally $\left.F_{\text {MEY }}<F_{\text {MSY }}\right)$.

The fishing patterns leading to the efficiency frontier with respect to profits and ecosystem state in the Baltic Sea are surprisingly similar to the patterns needed to reach the yield-ecosystem state efficiency frontier (Fig. 6), but with more moderate exploitation rates. The efficient fishing patterns include fishing all three species (sprat (Sprattus sprattus, Clupeidae), herring (Clupea harengus, Clupeidae) and cod (Gadus morhua, Gadidae)) in the system. In the case of the North Sea, the profitecosystem state frontier is achieved by exploiting medium and large fish very moderately, while maintaining lower exploitation on the small species. As the larger fish get more exploited, there is also the possibility to gain profit from catching small fish (sprat, sandeel (Ammodytes marinus, Ammodytidae), Norway pout (Trisopterus esmarkii, Gadidae) and herring).

\section{Discussion}

Here, we demonstrate the utility of Pareto efficiency as a concept for navigating multi-objective

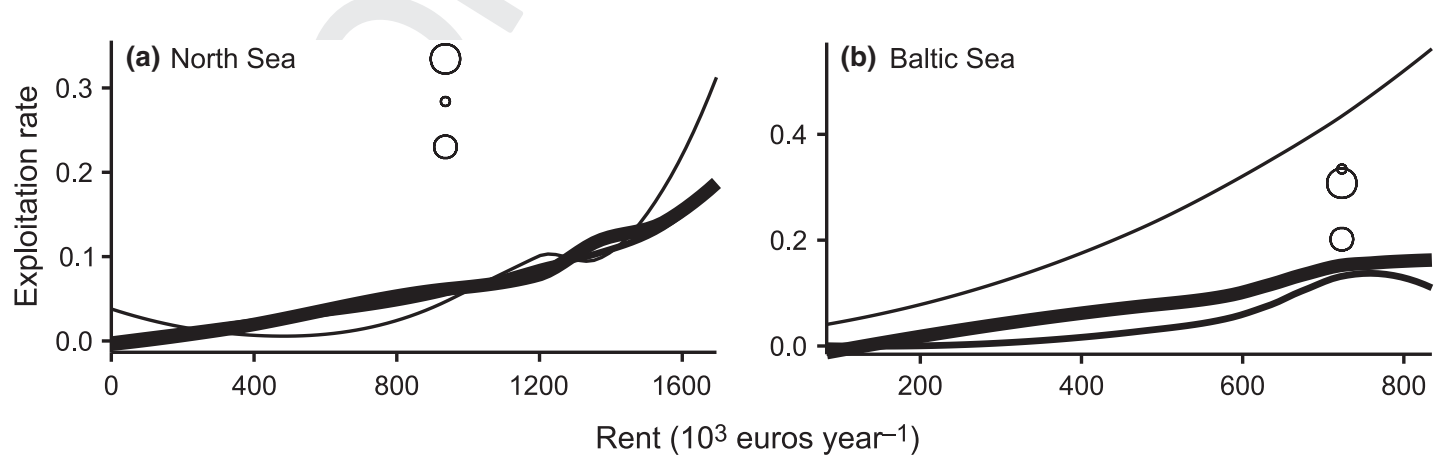

Figure 6 Exploitation rate (mean yield/biomass for small, large and medium species) used for the economic efficiency frontiers. Line thickness indicates fleets targeting small medium or large species. Circles indicate the equilibrium total yield of running 2010 exploitation (small, medium and large circle circumference denotes fleets of that size). 
trade-offs in ecosystem-based fisheries management. Efficiency frontiers can be directly applied to investigate the long-term efficiency of fishing patterns in aquatic ecosystems with respect to multiple objectives in systems with interacting species. We suggest that assessing Pareto efficiency with respect to key objectives as an essential part of a management strategy evaluation (Smith et al. 1999). Efficiency frontiers and trade-off analyses are already widely used in this manner in both terrestrial and marine spatial planning, for example Polasky et al. (2008) and Rassweiler et al. (2014).

We find positive trends over time in the ecosystem-level efficiency of fisheries, with respect to our measure of ecosystem state and both yield and profits, in all of the LMEs examined. With respect to yield and ecosystem state, ecosystem-level fishing patterns have either moved closer to the efficiency frontier (Baltic Sea, NEUSCS and Benguela Current, Fig. 3) or moved along the frontier towards better long-term ecosystem states and 2030\% lower long-term yields (North Sea and Barents Sea, Fig. 3). The former LMEs therefore have scope for further yield-ecosystem state win-wins, as they have not yet reached the efficiency frontier. We also find that the economic efficiency of both the North Sea and the Baltic Sea has been increasing over the last decade and now exhibits positive rent on the ecosystem scale, albeit with sizeable win-wins remaining.

Our results provide cause for optimism, like other recent studies finding evidence of improvements in the management of assessed stocks (Hilborn and Ovando 2014), although certainly not complacency. In particular, we find evidence for sizeable profit-conservation win-wins in the North Sea and Baltic Sea LMEs, which could be realized by fishing less and redistributing fishing pressure across species to better account for indirect effects of fishing across species. This line of reasoning has parallels to recent calls for more holistic fishing patterns: (i) 'balanced harvesting' (e.g. Zhou et al. 2010; Garcia et al. 2012) - fishing all ecosystem components in proportion to their productivity or (ii) protecting 'forage fish' to increase food web stability and predator yields (Smith et al. 2011; Pikitch et al. 2012; Essington and Munch 2014); so it is worth briefly highlighting the nuances.

First, the fishing patterns required to reach the efficiency frontier do not conform to a universal balanced pattern (Figs 4 and 6), and the economic analysis specifically targets some of issues related to balanced harvesting by accounting for size specific price differences (Burgess et al. 2015). Balanced harvesting could perhaps be an improvement from the status quo with respect to yield and conservation (Jacobsen et al. 2014; Zhou et al. 2014). Second, in contrast to some calls for balanced harvesting, we only analyse alternate fishing patterns among already commercially exploited species; thus, implementing recommendations arising from our analyses would not necessarily require a large change to the current management system or fishing technologies (e.g. Reid et al. 2016). Third, our models suggest in some LMEs that efficiency can be improved by slightly increasing exploitation on some forage fish (e.g. in the North Sea or the economic frontier in the Baltic Sea), in contrast to previous calls to reduce fishing on forage fish (e.g. Smith et al. 2011). This discrepancy may be system-specific and may in part be due to size-based models taking ontogenetic ecological changes (e.g. adult forage fish competing with juvenile predators, see e.g. Jacobsen et al. (2015) and Essington and Munch (2014)) into account that other ecosystem models do not, discussed in more detail below; in either case it merits further study. However, our results are in agreement with both balanced harvesting studies and studies suggesting the protection of forage fish in the general suggestion of adjusting ecosystem-level fishing patterns to better account for ecologically driven externalities across fisheries. Finally, we note that the cause for optimism found here does not necessarily extend to all exploited ecosystems. The examined sample is heavily biased towards fully exploited, well-managed systems.

We find that single-species MSY management, by failing to account for ecologically mediated indirect effects of fishing, is likely to perform inefficiently with respect to yields, economic rents and ecosystem impact. This is not a surprising result; it is well-known that fishing has important indirect effects in an ecosystem context, especially through food chains (Frank et al. 2005; Walters et al. 2005; Andersen et al. 2015a). Forage fish stocks, for example, provide significant biological and economic supporting services to higher-trophic-level fisheries (Smith et al. 2011; Pikitch et al. 2012; Plaganyi and Essington 2014). Conversely, depletion of species higher in the food chain can increase the yields of prey fisheries through 
predator release (Rice and Gislason 1996; Daan et al. 2005; Matsuda and Abrams 2006).

\section{Moving towards the frontier}

Efficiency frontier analyses not only identify what combinations of outcomes are possible, but also provide specific suggestions for how to get there. In our analysis, the direction of change in the fishing pattern projected to promote efficiency can be derived from Figs 4 and 6 (for yields and ecosystem impact, and economic rents and ecosystem impact, respectively). For example, our results suggest that efficiency could be increased in the North Sea and Baltic Sea, with respect to economic rents and ecosystem impact, by reducing fishing pressure on most stocks. In the Baltic Sea, economic efficiency is obtained with exploitation relatively higher on sprat than on other species, as sprat can interact with younger life stages of cod (a commercially valuable stock) through competition and predation (Van Leeuwen et al. 2008; Köster et al. 2009).

Because the recommendations of efficiency frontier analyses are almost always derived from models, it is also important to consider implementation challenges that are unaccounted for (and also to subject the recommendations to scrutiny from other lines of evidence). For example, in Polasky et al.'s (2008) analysis of land use for biodiversity and economic returns, many of the efficient landuse patterns would require moving large residential areas and other drastic and likely infeasible interventions. Often the practical utility of the efficiency frontier analysis is demonstrating the mere existence of win-wins and the general direction management needs to move in to realize them (which can motivate policymakers and stakeholders), rather than providing an accurate estimate of the magnitude of what can be realistically achieved. In our analysis, the notion of increasing fishing pressure on Baltic Sea sprat, for example, could be both scientifically and politically contentious, given that it is currently estimated (by single-species assessments) to be experiencing mild overfishing (although not overfished). Thus, this recommendation would likely (and rightly) be subjected to rigorous scrutiny and debate before being implemented. The recommendation to reduce fishing pressure on other stocks would likely be less scientifically contentious but could nonetheless face political barriers (Österblom et al. 2010).
Charles et al.(2015) and Reid et al. (2016) discuss similar implementation challenges in more detail in the context of implementing balanced harvesting. In general, implementing changes to ecosystem-level fishing patterns could face myriad technological challenges (especially in multispecies fisheries with non-selective gears) and institutional challenges, which merit consideration.

\section{Models and calibration}

The results should be interpreted in light of the simplifying assumptions within the model: (i) feeding interactions are determined by individual size only and not by species-specific preferences. The remarkably good performance of the calibrated model confirms that this assumption captures the major part of the actual interactions (Figure C1-C6). (ii) The models only resolves assessed stocks within a LME and thereby does not represent rare and potentially vulnerable species such as elasmobranchs captured as by-catch or in mixed fisheries (Stevens et al. 2000), which are of little commercial interest. These species are therefore not part of the ecological indicator and have to be considered separately; (iii) the bioeconomic model is calibrated such that the rent in the equilibrium state in the North Sea is close to zero following Andersen et al. (2015a). This calibration procedure does not give a precise estimate of the economic rent of the two systems, but it does not influence the direction and the distance to the economic frontier. The models applied here should be perceived as 'strategic ecosystem models' that aid in the long-term development of fishing patterns in ecosystems and act as a supplement to tactical assessment models (Collie et al. 2014).

\section{Applications and conclusions}

The implementation of ecosystem-based fisheries management has been discussed for decades, but it is evident that the path to get there is difficult (Skern-Mauritzen et al. 2015). One of the crucial steps to implementing EBFM is developing strategies that consider trophic interactions, climate and human impacts from a tactical perspective (Collie et al. 2014; Plagányi et al. 2014). Instead of proposing a strategy a priori that should guide fisheries management efficiently, the methods presented here provide a framework to investigate which strategies optimize desired objectives and 
what the associated trade-offs are. All objectives cannot be optimized simultaneously, as some objectives will be inherently conflicting (Link 2002; Andersen et al. 2015a).

We suggest using Pareto efficiency as a concept to guide management of exploited populations with conflicting objectives. The framework presented here emphasizes that the challenge of weighing objectives against one another does not have to impede consensus or progress as long as win-wins exist. In many of the cases in which the Pareto framework has been used - here included - available win-wins have been found to be common.

\section{Acknowledgements}

NSJ and KHA are funded by the Centre for Ocean Life: A Villum Kann Rasmussen Centre of Excellence funded by the Villum Foundation. KHA is further support by the BIOC3 EU FP7 project. MGB is supported by the Waitt Foundation and the Ocean Conservancy. We thank Cody Szuwalski for comments on an early version of the paper. Model calibrations and $\mathrm{R}$ code are available upon request.

\section{References}

Andersen, K.H., Brander, K. and Ravn-Jonsen, L. (2015a) Trade-offs between objectives for ecosystem management of fisheries. Ecological Applications 25, 1390-1396.

Andersen, K.H., Jacobsen, N.S. and Farnsworth, K.D. (2015b) The theoretical foundations for size spectrum models of fish communities. Canadian Journal of Fish-

6 eries and Aquatic Science, ???, ???-??? in review.

Brown, J.H., Gillooly, J.F., Allen, A.P., Savage, V.M. and West, G.B. (2004) Toward a metabolic theory of ecology. Ecology 85, 1771-1789.

Burgess, M.G., Diekert, F.K., Jacobsen, N.S., Andersen, K.H. and Gaines, S.D. (2015) Remaining questions in the case for balanced harvesting. Fish and Fisheries, ???,

7 ???-??? n/a-n/a.

Cardinale, B.J., Duffy, J.E., Gonzalez, A. et al. (2012) Corrigendum: Biodiversity loss and its impact on humanity. Nature 489, 326-326.

Carpenter, S.R., Mooney, H. A., Agard, J. et al. (2009) Science for managing ecosystem services: Beyond the Millennium Ecosystem Assessment. Proceedings of the National Academy of Sciences of the United States of America 106, 1305-1312.

Charles, A., Garcia, S.M. and Rice, J.C. (2015) Balanced harvesting in fisheries: economic considerations. ICES Journal of Marine Science 69, 380-388.
Cheung, W.W.L. and Sumaila, U.R. (2008) Trade-offs between conservation and socio-economic objectives in managing a tropical marine ecosystem. Ecological Economics 66, 193-210.

Collie, J.S., Botsford, L.W., Hastings, A. et al. (2014) Ecosystem models for fisheries management: finding the sweet spot. Fish and Fisheries ???, 1-25.

Daan, N., Gislason, H., Pope, J.G. and Rice, J.C. (2005) Changes in the North Sea fish community: evidence of indirect effects of fishing? ICES Journal of Marine Science: Journal du Conseil 62, 177-188.

Essington, T. and Munch, S. (2014) Trade-offs between supportive and provisioning ecosystem services of forage species in marine food webs. Ecological Applications 24, 1543-1557.

Frank, K.T., Petrie, B., Choi, J.S. and Leggett, W.C. (2005) Trophic Cascades in a Formerly Cod-Dominated Ecosystem. Science 308, 1621-1623.

Garcia, S.M., Kolding, J., Rice, J. et al. (2012) Reconsidering the Consequences of Selective Fisheries. Science 335, 1045-1047.

Halpern, B.S., Klein, C.J., Brown, C.J. et al. (2013) Achieving the triple bottom line in the face of inherent trade-offs among social equity, economic return, and conservation. Proceedings of the National Academy of Sciences of the United States of America 110, 6229-6234.

Hartvig, M., Andersen, K.H. and Beyer, J.E. (2011) Food web framework for size-structured populations. Journal of Theoretical Biology 272, 113-122.

Hilborn, R. and Ovando, D. (2014) Reflections on the success of traditional fisheries management. ICES Journal of Marine Science 71, 1040-1046.

Hilborn, R., Fulton, E.A., Green, B.S., Hartmann, K., Tracey, S.R. and Watson, R.A. (2015) When is a fishery sustainable? ????? 9, 1-9.

Jacobsen, N.S., Gislason, H. and Andersen, K.H. (2014) The consequences of balanced harvesting of fish communities. Proceedings. Biological sciences/The Royal Society 281, 20132701.

Jacobsen, N.S., Essington, T.E. and Andersen, K.H. (2015) Comparing model predictions for ecosystem based management. Canadian Journal of Fisheries and Aquatic Sciences. ???, ???-???.

Köster, F.W., Vinther, M., Mackenzie, B.R., Eero, M. and Plikshs, M. (2009) Environmental Effects on Recruitment and Implications for Biological Reference Points of Eastern Baltic Cod (Gadus morhua). Journal of Northwest Atlantic Fishery Science 41, 205-220.

Lester, S.E., McLeod, K.L., Tallis, H. et al. (2010) Science in support of ecosystem-based management for the US West Coast and beyond. Biological Conservation 143, $576-587$.

Lester, S.E., Costello, C., Halpern, B.S., Gaines, S.D., White, C. and Barth, J.A. (2013) Evaluating tradeoffs among ecosystem services to inform marine spatial planning. Marine Policy 38, 80-89. 
Link, J.J.S. (2002) What does ecosystem-based fisheries management mean. Fisheries (Bethesda) 27, 18-21.

Matsuda, H. and Abrams, P.A. (2006) Maximal Yields from Multispecies Fisheries Systems : Rules for Systems with Multiple Trophic Levels. America 16, 225-237.

Odum, E.P., Odum, H.T. and Andrews, J. (1971) Fundamentals of Ecology, Vol. 3. Saunders, Philadelphia.

Österblom, H., Gårdmark, A., Bergström, L. et al. (2010) Making the ecosystem approach operational-Can regime shifts in ecological- and governance systems facilitate the transition? Marine Policy 34, 1290-1299.

Patrick, W.S. and Link, J.S. (2015) Hidden in plain sight: Using optimum yield as a policy framework to operationalize ecosystem-based fisheries management. Marine Policy 62, 74-81.

Pikitch, E., Santora, E., Babcock, A. et al. (2004) Ecosystem-based fishery management. Science (New York, N.Y.) 305, 346-347.

Pikitch, E.K., Rountos, K.J., Essington, T.E. et al. (2012) The global contribution of forage fish to marine fish-

11 eries and ecosystems. Fish and Fisheries ???, ???-???

Plaganyi, E.E. and Essington, T.E. (2014) When the SURFs up, forage fish are key. Fisheries Research 159 , 68-74.

Plagányi, É.E., Punt, A.E., Hillary, R. et al. (2014) Multispecies fisheries management and conservation: tactical applications using models of intermediate complexity. Fish and Fisheries 15, 1-22.

Polasky, S., Nelson, E., Lonsdorf, E., Fackler, P. and Starfield, A. (2005) Conserving species in a working landscape: land use with biological and economic objectives. Ecological Society of America 15, 13871401.

Polasky, S., Nelson, E., Camm, J. et al. (2008) Where to put things? Spatial land management to sustain biodiversity and economic returns. Biological Conservation 141, 1505-1524.

Rassweiler, A., Costello, C., Hilborn, R. and Siegel, D. A. (2014) Integrating scientific guidance into marine spatial planning Integrating scientific guidance into mar-

12 ine spatial planning. ???? ???, ???-???

Reid, D.G., Graham, N., Suuronen, P., He, P. and Pol, M. (2016) Implementing balanced harvesting: practical challenges and other implications. ICES Journal of Mar-

13 ine Science ???, ???-???

Ricard, D., Minto, C., Jensen, O.P. and Baum, J.K. (2011) Examining the knowledge base and status of commercially exploited marine species with the RAM Legacy Stock Assessment Database. Fish and Fisheries ???,

14 ???-??? no-no.

Rice, J. and Duplisea, D. (2014) Management of fisheries on forage species: the test-bed for ecosystem approaches to fisheries. ICES Journal of Marine Science 71, 143-152.

Rice, J. and Gislason, H. (1996) Patterns of change in the size spectra of numbers and diversity of the North
Sea fish assemblages, as reflected in surveys and models. ICES Journal of Marine Science 53, 1214-1225.

Skern-Mauritzen, M., Ottersen, G., Handegard, N.O. et al. (2015) Ecosystem processes are rarely included in tactical fisheries management. Fish and Fisheries ???, ???-??? n/a-n/a.

Smith, A.D., Sainsbury, K.J. and Stevens, R.A. (1999) Implementing effective fisheries-management systems management strategy evaluation and the Australian partnership approach. ????, ???, 967-979.

Smith, A.D.M., Brown, C.J., Bulman, C.M. et al. (2011) Impacts of fishing low-trophic level species on marine ecosystems. Science (New York, N.Y.) 333, 1147-1150.

Stevens, J.D., Bonfil, R., Dulvy, N.K. and Walker, P.A. (2000) The effects of fishing on sharks, rays, and chimaeras (chondrichthyans), and the implications for marine ecosystems. ICES Journal of Marine Science: Journal du Conseil 57, 476-494.

Ursin, E. (1973) On the prey size preferences of cod and dab. Meddelelser fra Danmarks Fiskeri- og Havundersogelser 7, 85-98.

Van Leeuwen, A., De Roos, A.M. and Persson, L. (2008) How cod shapes its world. Journal of Sea Research 60, 89-104.

Walters, C.J., Christensen, V., Martell, S.J. and Kitchell, J.F. (2005) Possible ecosystem impacts of applying MSY policies from single-species assessment. ICES Journal of Marine Science 62, 558-568.

White, C., Halpern, B.S. and Kappel, C.V. (2012) Ecosystem service tradeoff analysis reveals the value of marine spatial planning for multiple ocean uses. Proceedings of the National Academy of Sciences 109, 4696-4701.

Zhou, S., Smith, A.D.M., Punt, A.E. et al. (2010) Ecosystem-based fisheries management requires a change to the selective fishing philosophy. Proceedings of the National Academy of Science U.S.A. 107, 9485-9489.

Zhou, S., Smith, A.D. and Knudsen, E.E. (2014) Ending overfishing while catching more fish. Fish and Fisheries ???, ???-???, n/a-n/a.

\section{Supporting Information}

Additional Supporting Information may be found in the online version of this article:

Appendix S1. Content

Table A1. List of parameters. *see Appendix B.

Figure C1. Temporal spawner biomass (metric tons) from the North Sea.

Figure C2. Temporal spawner biomass (metric tons) from the Baltic Sea.

Figure C3. Temporal spawner biomass (metric tons) from the Benguela Current.

Figure C4. Temporal spawner biomass (metric tons) from the Barents Sea. 
Figure C5. Temporal spawner biomass (metric tons) from the North East US Continental Shelf (see remaining species In Figure A6).

Figure C6. Temporal spawner biomass (metric tons) from the North East US Continental Shelf.

Figure D1. North Sea calibration verification under equilibrium.

Figure D2. Baltic Sea Calibration. (a-l) see Figure D1 caption.

Figure D3. Benguela Current calibration. (a-l) see Figure D1 caption.
Figure D4. Barents Sea calibration. (a-l) see Figure D1 caption.

Figure D5. North East US Continental Shelf calibration. (a-l) see Figure D1 caption.

Figure E1. The profit divided by the cost as a function of asymptotic weight under the equilibrium calibrations. (a) The North Sea, (b) The Baltic Sea.

Figure G1. Sensitivity of the yield-ecology efficiency frontiers to changes in $\tau$ (both axes scaled to 1 ). 


\section{Author Query Form}

Journal: $\quad$ FAF

Article: $\quad 12171$

Dear Author,

During the copy-editing of your paper, the following queries arose. Please respond to these by marking up your proofs with the necessary changes/additions. Please write your answers on the query sheet if there is insufficient space on the page proofs. Please write clearly and follow the conventions shown on the attached corrections sheet. If returning the proof by fax do not write too close to the paper's edge. Please remember that illegible mark-ups may delay publication.

Many thanks for your assistance.

\begin{tabular}{|c|c|c|}
\hline Query reference & Query & Remarks \\
\hline 1 & $\begin{array}{l}\text { AUTHOR: Two article title has been presented in author } \\
\text { file. Please check and approve whether we have } \\
\text { followed correct one. }\end{array}$ & \\
\hline 2 & $\begin{array}{l}\text { AUTHOR: Please confirm that given names (red) and } \\
\text { surnames/family names (green) have been identified } \\
\text { correctly. }\end{array}$ & \\
\hline 3 & $\begin{array}{l}\text { AUTHOR: Please provide fax number for corresponding } \\
\text { author and also check the corresponding author details. }\end{array}$ & \\
\hline 4 & $\begin{array}{l}\text { AUTHOR: The phrases "North East US Continental Shelf" } \\
\text { and "Northeast US Continental Shelf" have been used } \\
\text { inconsistently throughout the article. Kindly suggest } \\
\text { which has to be followed. }\end{array}$ & \\
\hline 5 & $\begin{array}{l}\text { AUTHOR: The term 'F.i', '0.i', } \infty . i \text {, and i.o has been } \\
\text { changed to 'F,i', '0,i', } \infty, i \text { and } i, 0 \text {. Please check and } \\
\text { correct if necessary. }\end{array}$ & \\
\hline 6 & $\begin{array}{l}\text { AUTHOR: Please provide the volume number, page } \\
\text { range for reference Andersen et al. }(2015 \mathrm{~b}) \text {. }\end{array}$ & \\
\hline 7 & $\begin{array}{l}\text { AUTHOR: Please provide the volume number, page } \\
\text { range for reference Burgess et al. }(2015) \text {. }\end{array}$ & \\
\hline 8 & $\begin{array}{l}\text { AUTHOR: Please provide the volume number for } \\
\text { reference Collie et al. (2014). }\end{array}$ & \\
\hline 9 & $\begin{array}{l}\text { AUTHOR: Please provide the journal title for reference } \\
\text { Hilborn et al. (2015). }\end{array}$ & \\
\hline 10 & $\begin{array}{l}\text { AUTHOR: Please provide the volume number, page } \\
\text { range for reference Jacobsen et al. }(2015) \text {. }\end{array}$ & \\
\hline 11 & $\begin{array}{l}\text { AUTHOR: Please provide the volume number, page } \\
\text { range for reference Pikitch et al. (2012). }\end{array}$ & \\
\hline
\end{tabular}




\begin{tabular}{|l|l|l|}
\hline 12 & $\begin{array}{l}\text { AUTHOR: Please provide the journal title, volume } \\
\text { number, page range for reference Rassweiler et al. } \\
(2014) .\end{array}$ & $\begin{array}{l}\text { AUTHOR: Please provide the volume number, page } \\
\text { range for reference Reid et al. (2016). }\end{array}$ \\
\hline 13 & $\begin{array}{l}\text { AUTHOR: Please provide the volume number, page } \\
\text { range for reference Ricard et al. (2011). }\end{array}$ & \\
\hline 14 & $\begin{array}{l}\text { AUTHOR: Please provide the volume number, page } \\
\text { range for reference Skern-Mauritzen et al. (2015). }\end{array}$ & \\
\hline 16 & $\begin{array}{l}\text { AUTHOR: Please provide the journal title, volume } \\
\text { number for reference Smith et al. (1999). }\end{array}$ & \\
\hline 17 & $\begin{array}{l}\text { AUTHOR: Please provide the volume number, page } \\
\text { range for reference Zhou et al. (2014). }\end{array}$ & $\begin{array}{l}\text { AUTHOR: Supplementary Material/Supporting } \\
\text { Information has been supplied with this paper. Please } \\
\text { check the legends to ensure they are correct. }\end{array}$ \\
\hline 18 &
\end{tabular}

